\title{
RISORGIMENTO E MODERNITÀ DAI CONGRESSI DEGLI SCIENZIATI ALLE ESPOSIZIONI POSTUNITARIE
}

\author{
CARLO G. LACAITA (*)
}

SunTO. - Nel corso dell'Ottocento sia i Congressi degli scienziati che le Esposizioni industriali contribuirono alla presa di coscienza collettiva dei cambiamenti dovuti all'avanzamento tecnico-scientifico e alla diffusione dell'industrializzazione e della modernizzazione più in generale. Il saggio esamina il nesso che si stabilì, sia prima che dopo il 1861, fra questi aspetti della modernità e il moto risorgimentale, e pone in rilievo come insieme all'unità e all'indipendenza politica della nazione gli uomini del Risorgimento perseguirono l'inserimento dell'Italia nella "civiltà moderna" e la sua partecipazione attiva ai grandi "progressi del secolo".

$* * *$

ABSTRACT. - During the 19. Century both the scientific congresses and the industrial exhibitions contributed to the collective consciousness-raising of the changes due to the technical-scientific progress and the diffusion of the industrialization and modernization in wider terms. This paper analyzes the connection, both before than after 1861, between these aspects of modernity and the Risorgimento movement, and highlights how, together with the unity and political indipendence of the country, the men of Risorgimento pursued the integration of Italy in the "modern civilization" and its active participation to the big "progresses of the century".

(*) Università degli Studi di Milano, Italia. E-mail: carlo.lacaita@virgilio.it 
1. Si è molto scritto sui congressi degli scienziati che per nove anni di seguito, dal 1839 al 1847, si tennero in altrettante città italiane. Molto meno si è detto delle esposizioni di prodotti e di strumenti, che sempre in quegli anni furono organizzate in varie sedi dopo le rassegne allestite dall'inizio dell'Ottocento sull'esempio delle mostre d'oltralpe. La diversa attenzione è certamente legata agli orientamenti storiografici, in particolare della storiografia risorgimentale, rimasta a lungo influenzata dall'indirizzo etico-politico, che privilegiava la storia dello stato, delle élites politiche, delle loro idee e delle loro lotte, a scapito di altri aspetti del secolo XIX, da quelli economici e sociali a quelli tecnico-scientifici e agli altri, via via presi in considerazione in una visione sempre più ampia e articolato del periodo e dei processi che lo attraversarono.

Proprio in quanto connessi a questi processi e alla volontà di farne parte, le esposizioni e i congressi rispecchiarono esigenze comuni e insieme differenti perché legate ad aspetti e a fasi storiche diversi. Se le prime esposizioni apparvero nell'Inghilterra che stava iniziando la rivoluzione industriale, per passare in Francia e altrove, al seguito delle armate francesi (in Italia le prime si ebbero a Torino e a Milano nel 1805 in occasione dell'incoronazione di Napoleone a re d'Italia, e proseguirono anche dopo, nonostante le intenzioni dei regimi restaurati di chiudere col passato rivoluzionario), i Congressi degli scienziati nacquero dopo la restaurazione al di là delle Alpi ${ }^{1}$ e si affermarono in Italia quando la rivoluzione industriale, giunta a maturazione in Inghilterra, aveva coinvolto vari paesi e posto a tutti il problema del divario e di come colmarlo. Di fronte all'accumularsi delle innovazioni, ai telai e ai filatoi automatici mossi dalle macchine a vapore, alle locomotive ferroviarie e alle nuove turbine idrauliche, all'illuminazione a gas e ai telegrafi elettrici, anche nell'Italia degli anni Trenta e Quaranta emerse il tema dei ritardi rispetto ai paesi più progrediti e dei rimedi da adottare per non restare ai margini dello sviluppo.

Era a questo che pensava Cattaneo quando, nel 1837, illustrava su "L'Eco della Borsa" la straordinaria capacità produttiva delle imprese tessili di Birmingham e affermava subito dopo che non si poteva in alcun

1 Fu il naturalista e medico tedesco Lorenz Oken, direttore della rivista "Isis", a promuovere nel 1821 il primo congresso scientifico che si tenne a Lipsia nel 1822, dando il via a un movimento che si diffuse in altri paesi fra cui l'Italia. 
modo "né gareggiare né resistere a quella forza industriale se non coll' $i$ mitarla". A porsi il problema non era solo un intellettuale di razza come il pubblicista lombardo, dal 1839 impegnato nella conduzione di un repertorio intitolato "Il Politecnico". In termini analoghi si esprimevano sia Carlo Ignazio Giulio, docente di meccanica a Torino e consigliere di Stato, quando affermava: "Nelle presenti condizioni della civiltà, l'industria di ogni paese non ha altra alternativa che questa: abbracciare $\mathrm{i}$ moderni perfezionamenti, oppure languire e perire""; sia Luigi Alessandro Parravicini, pedagogista e dirigente a Venezia di una delle prime scuole tecniche istituite in Italia, quando al quinto Congresso degli scienziati italiani si chiedeva: "Chi in Italia sa applicare il gas illuminante? Chi la forza gigantesca del vapore alle arti? Chi sa costruire le macchine più utili alle manifatture del lino e del cotone?" E rispondeva: "Pochi forestieri; e più pochi de' nostri", per concludere subito dopo che occorreva diffondere i nuovi saperi fra gli italiani per supportare "colla scienza, in questa universale gara di produzioni, le speculazioni del manifattore, del commerciante, dell'agricoltore" ${ }^{4}$. Sempre negli anni dei congressi scientifici il giornalista milanese Michele Battaglia, con riferimento proprio al nesso tra esposizioni e innovazioni produttive, metteva in rilievo nell'"Eco della Borsa" l'essenza stessa del processo di modernizzazione in atto, affermando che, se le esposizioni mostravano prodotti sempre diversi, era perché seguivano la "legge di questo secolo di movimento, pel quale l'immobilità" di fatto equivaleva a "decaden$z^{\prime \prime}{ }^{5}$. Né da diverse considerazioni fu mosso il fisico Giò Alessandro Majocchi, assiduo frequentatore dei congressi scientifici, quando decise di dar vita agli "Annali di fisica, chimica e matematiche" col proposito di incrementare fra i congressisti di ogni parte d'Italia il confronto e lo scambio collaborativo sia sul piano della ricerca che su quello delle

2 C. Cattaneo, Scritti scientifici e tecnici, a cura di C.G. Lacaita, I. 1823-1848, Firenze, Giunti-Barbera, 1969, p. 72.

3 C.I. Giulio, Giudizio della R. Camera di agricoltura e commercio di Torino e notizie sulla patria industria, Torino, Stamperia reale, 1844, p. 117.

4 L.A. Parravicini, Rapporto sulle scuole tecniche del Regno Lombardo-Veneto e specialmente sulla scuola tecnica di Venezia, in Atti del V Congresso degli Scienziati, Lucca, 1843, pp. 164-165.

5 M. Battaglia, Rapporto all'adunanza generale della Società d'incoraggiamento, 1844, ora in Appendice a S. Levati, Giornalismo e tutela degli interessi mercantili. Michele Battaglia (1800-1870), Soveria Mannelli, Rubbettino, 1999, p. 177. 
applicazioni pratiche e produttive. Agli articoli scientifici degli "Annali", infatti, non tardò ad affiancare un apposito "Bollettino dell'industria meccanica e chimica" volto a divulgare fra i produttori italiani i ritrovati tecnici e i procedimenti innovativi ${ }^{6}$.

2. Già nella prima fase della Restaurazione l'interesse per "le conquiste della civiltà" e la loro introduzione nella penisola si era manifestato nelle iniziative dei Confalonieri e dei Porro Lambertenghi, dalle scuole di mutuo insegnamento all'introduzione dell'illuminazione a gas e della navigazione a vapore, al progetto di un "Ateneo politecnico" destinato a ospitare conferenze, letture, corsi d'istruzione tecnica, la borsa e altro ancora ${ }^{7}$. E se la dura repressione dei primi moti risorgimentali non mancò di colpire anche le iniziative modernizzatrici, le istanze di cambiamento non tardarono a riproporsi attraverso la crescente produzione di carta stampata, almanacchi, fogli di "cognizioni utili", giornali e riviste i cui titoli (si pensi alle testate del gruppo Lampato, che andavano dagli "Annali universali di statistica, economia pubblica, storia, viaggi e commercio", agli "Annali di medicina”, dagli "Annali di tecnologia, agricoltura, arti e mestieri", a quelli di "farmacia e chimica" ${ }^{\prime}$ ) erano già tutto un programma.

Ciò che distingue il secondo ventennio dell'Ottocento dal primo è che, con l'intensificarsi delle innovazioni, a preoccuparsi e a muoversi non sono solo gli ambienti più avveduti della società civile, ma anche le componenti più riflessive dei regimi assoluti, che ottennero dai sovrani restaurati una serie di aperture come l'invio all'estero di funzionari ed esperti col compito di studiare le novità da mutuare, l'introduzione nelle scuole di testi più aggiornati, il riordino di istituzioni culturali, la

6 G.A. Majocchi, Dello studio delle scienze applicate all'industria, Fontana, Milano, 1832. Su questa figura ancora poco studiata mi sia consentito rinviare al mio Un organizzatore della cultura scientifica e tecnica italiana nell' età del Risorgimento: Gio. Alessandro Majocchi e gli «Annali di fisica, chimica e matematiche», in AA.VV., Ricerche di storia in onore di Franco Della Peruta. a cura di M.L. Betri e D. Bigazzi, vol. II, Milano, Angeli, 1996, pp. 198-218.

7 Cfr. F. Della Peruta, Conservatori, liberali e democratici nel risorgimento, Milano, Angeli, 1989, pp. 27-60.

8 Su Lampato v. S. La Salvia, Giornalismo lombardo: gli "Annali universali di statistica" (1824-1844), Roma, Elia, 1977 e M. Berengo, Intellettuali e librai nella Milano della Restaurazione, Torino, Einaudi, 1980, ad ind. 
possibilità di organizzare sia le esposizioni industriali sia i congressi scientifici, che riunendo centinaia di intellettuali di vari stati e regioni comportavano pure rischi e suscitavano preoccupazioni.

In questa direzione si mosse la decisione di Vienna di fare dell'incoronazione di Ferdinando I a re del Lombardo-Veneto, nel 1838, l'occasione per varare insieme a un'amnistia generale per i condannati politici, una serie di provvedimenti capaci di migliorare i rapporti con i ceti intellettuali e produttivi sul terreno della modernizzazione. Fu così riorganizzato l'Istituto di scienze, lettere ed arti' e furono attivate le prime scuole tecniche nelle due capitali del Lombardo-Veneto; si consentì la costituzione della Società per la ferrovia Milano-Venezia e si autorizzò la nascita del Museo civico di storia naturale, proposta da Giuseppe De Cristoforis e da Giorgio Jan con la cessione delle loro raccolte alla città di Milano. Di più. Fu accolto il progetto di un'associazione impegnata a promuovere il miglioramento dei sistemi produttivi, elaborato presso la Camera di Commercio di Milano da un gruppo di commercianti e manifatturieri riunitisi attorno a Enrico (o Heinrich) Mylius, imprenditore di origine tedesca e di formazione europea. L'approvazione dello statuto del nuovo ente però fu tutt'altro che rapida, a conferma delle tenaci resistenze interne al governo di Vienna verso la concessione di autonomia effettiva alle forze sociali. Così come invece la pronta adesione dei milanesi alle iniziative del nuovo sodalizio mostra quanto gli ambienti più dinamici fossero pronti a sostenerle.

9 Il nuovo regolamento organico affermava testualmente: "Lo scopo dell'Istituto è di promuovere quegli studi che hanno immediata e principale influenza sulla prosperità e sulla coltura scientifica generale delle scienze nelle provincie componenti il Regno lombardo-veneto. Sono quindi oggetto delle cure e degli studj dell'Istituto tutte le scienze che tendono a promuovere l'agricoltura, le arti e il commercio, non meno che le lettere nel più lato senso della parola, siccome quelle che costituiscono il fondamento principale per portare ad un grado elevato la coltura generale". Oltre che i premi d'industria, l'Istituto ebbe il compito di organizzare un concorso "per la migliore soluzione d'un quesito scientifico" e di far conoscere strumenti di lavoro e di produzione nuovi riuniti in un apposito gabinetto tecnologico. Sulla riorganizzazione del 1838 cfr. F. Della Peruta, Coltura e organizzazione del sapere nella Lombardia dell'Ottocento. L'Istituto Lombardo di Scienze e Lettere dalla fondazione all'unità d'Italia, in L'Istituto Lombardo Accademia di Scienze e Lettere (secoli XIX-XX), I. Storia istituzionale, a cura di A. Robbiati Bianchi, Milano, Istituto Lombardo Accademia di Scienze e Lettere - Libri Scheiwiller, 2007. 
Dopo aver sperimentato la linea praticata dall'Istituto lombardo dei premi di industria a produttori e operai distintisi nel loro campo di attività, la Società d'incoraggiamento d'arti e mestieri, come presto si chiamò la nuova associazione, passò a promuovere specialmente la diffusione delle conoscenze più richieste dalle incalzanti innovazioni del periodo, secondo ciò che dal '39 sosteneva il "Politecnico" di Cattaneo. Il primo concreto passo nella nuova direzione fu la nascita della scuola di Chimica industriale, resa possibile da Antonio Kramer, o De Kramer, assiduo collaboratore del repertorio cattaneano oltre che membro dell'Istituto lombardo, che trasferì presso la Società d'incoraggiamento il proprio laboratorio privato ricco di attrezzature non comuni ${ }^{10}$.

Accanto a questa prima scuola tecnologico-sperimentale, resa subito nota per aver ospitato gli iscritti al sesto Congresso degli scienziati per assistere agli esperimenti di Boutigny d'Evreux e di Schönbein di Basilea, furono presto attivati altri corsi, da quello di fisica industriale affidato a Luigi Magrini, a quello di geometria descrittiva e di meccanica tenuto da Paolo Jacini, a quello di setificio svolto da Angelo Piazza e replicato dallo stesso docente a Como, il cui distretto serico era già uno dei più importanti d'Italia. Contemporaneamente furono messe a disposizione del pubblico una raccolta di modelli e disegni tecnologici, una biblioteca specializzata, un'officina capace di riprodurre meccanismi industriali, e varie commissioni di esperti per lo studio di specifici problemi.

Se la molteplicità degli obiettivi e la capacità di mobilitare attorno ad essi gruppi diversi suscitavano negli apparati di controllo sospetti e apprensione, l'indirizzo industrialista della Società destava obiezioni fra i più legati all'economia tradizionale, così come l'intenzione di aggregare tutte le province lombarde alle iniziative metropolitane della Società si scontrò con il timore di molti enti locali di perdere parte delle loro prerogative.

Consapevole dei passi avanti compiuti fin dal Settecento dall'agricoltura lombarda sia nell'area irrigua che in quella asciutta e collinare, la dirigenza della Società riteneva che, accanto alle attività rurali o strettamente legate ad esse, dalla lavorazione serica alla lattiero-casearia, fos-

10 Sul primo corso tenuto da De Kramer nel biennio 1844-45 v. ora A. De Kramer, Lezioni di chimica industriale 1844-1845, a cura di C.G. Lacaita, Milano, Federazione Nazionale del Cavalieri del Lavoro Gruppo Lombardo, 2012. 
sero da ammodernare ed estendere le attività manifatturiere e commerciali per potersi agganciare davvero ai cambiamenti d'oltralpe.

Intervenendo come relatore della Società Cattaneo indicava pertanto tra le mete da raggiungere la diffusione "delle vie ferrate" e dell'illuminazione a gas, il passaggio "dalla filatura del cotone alle più complicate fogge della sua tessitura", l'affinamento della "pratica di tingere ed imprimere le sete", la produzione meccanica del lino e della lana, una migliore utilizzazione dell'energia idraulica disponibile, l'ampliamento dei trasporti su acqua, con l'apertura dell'"aurea diagonale" destinata a congiungere "il Mincio al Verbano" e "l'Adriatico alla valle del Reno" ${ }^{11}$, con la prospettiva di ulteriori ampliamenti verso i Paesi Bassi e l'Inghilterra da una parte, e verso l'intero Mediterraneo dall'altra. E che tali indicazioni fossero condivise dalle forze sociali più dinamiche della regione, è provato dalla petizione preparata dallo stesso Cattaneo e presentata alle autorità da un gruppo di imprenditori aderenti alla Società d'incoraggiamento in favore della libertà di movimento sul Po e sul Lago Maggiore, impedita dai confini e dai vincoli doganali e daziari esistenti ${ }^{12}$. Di questi e di altri ostacoli si parlava di continuo nei congressi scientifici, sia che si discutesse della carta geologica nazionale, sia che si proponesse una statistica clinica comparata degli ospedali italiani, o "un Erbario generale italiano", o un "dizionario tecnologico" comune, o "una farmacopea uniforme italiana”, o si ragionasse ancora di come realizzare una rete ferroviaria integrata e un'unione doganale simile allo Zollverein tedesco, o di come passare dalle piccole rassegne locali all'Esposizione generale dell'industria nazionale, in cui i produttori italiani potessero finalmente conoscersi e coordinarsi ${ }^{13}$. Idea questa che pure si cercò di mettere in atto nel 1847, in occasione del nono Congresso di Venezia, ma con risultati molto scarsi per le difficoltà di spostarsi da uno stato all'altro e da una provincia all'altra.

Proprio per superare le chiusure localistiche Cattaneo aveva pro-

11 C. Cattaneo, Scritti economici, a cura di A. Bertolino, Firenze, Le Monnier, 1956, vol. III, p. 12.

12 Cfr. l'ultima delle Memorie di economia publica, che Cattaneo riunì con altre 14 nel 1860. Il volume cattaneano è stato ripubblicato in edizione anastatica dalla Banca del Monte di Milano nel 1983.

13 Così Pasquale Stanislao Mancini a Genova nel 1846, in Atti della ottava riunione degli scienziati italiani tenuta in Genova dal XIV al XXIX settembre MDCCCXLVI, 1857, p. 142. 
posto alle città sedi di congresso, di non insistere con i soliti libri di taglio municipalistico (spesso "vuoti e frivoli"), e di passare invece alla realizzazione di più ampie monografie regionali in grado di illuminare la variegata realtà italiana in una visione d'insieme e in una prospettiva di lunga durata. $\mathrm{E}$ in occasione del Congresso milanese del '44, avendo precisato ulteriormente la sua idea alla Municipalità milanese senza vederla accolta, riunì attorno al suo progetto le necessarie competenze e passò alla preparazione delle Notizie naturali e civili su la Lombardia, di cui tuttavia poté pubblicare solo il primo volume ${ }^{14}$.

Alle diverse monografie regionali, affermava Cattaneo, illustrando ai lettori il suo programma, si sarebbero dovuti far seguire "supplementi annui" anche di minor mole, con cui ampliare la conoscenza tanto delle "singole patrie municipali", quanto della "patria comune". In tal modo si sarebbero messe a fuoco sia l'identità "commista" del "colosso italiano" che le peculiarità delle sue regioni, segnate dall'intreccio plurisecolare fra le attitudini naturali dei diversi territori e le fatiche, gli atti di intelligenza, le creazioni culturali e le istituzioni civili depositatevi dalle popolazioni. Aggiungeva infine, rimarcando il nesso tra la conoscenza e la prassi, che, solo ottenendo "quell'intima e verace cognizione" del tutto e delle parti, del passato e del presente, si sarebbe potuto davvero "pensare e operare il pubblico bene entro i confini del possibile e dell'opportuno, e senza mistura di mali" ${ }^{15}$.

3. Più aumentava l'adesione ai progetti innovativi e con essa la convinzione che, per mettersi al passo con i "progressi del secolo", accorresse intensificare il moto riformatore, più gli innovatori incalzavano, ritenendo che si potesse ormai passare a cambiamenti via via più ampi e incisivi. Eloquente al riguardo è la vicenda del progetto di riforma del pubblico insegnamento, che nell'ambito dell'Istituto lombardo di scienze, lettere ed arti fu elaborato da un'apposita Commissione di esperti all'inizio del $1848^{16}$. Dando forma organica alle richieste avanza-

14 C. Cattaneo, Notizie naturali e civili su la Lombardia, ora riapparse nella nuova Edizione nazionale delle opere di Carlo Cattaneo promossa dal Comitato italosvizzero, vol. III, t. I, a cura di G. Bigatti, Firenze-Bellinzona, Le Monnier-Edizioni Casagrande, 2014.

15 C. Cattaneo, Scritti storici e geografici, a cura di G. Salvemini e E. Sestan, vol. I, Firenze, Le Monnier, 1957, p. 328.

16 Per il testo del progetto, stilato da Cattaneo in qualità di relatore della 
te negli ultimi tempi ${ }^{17}$, il progetto finì per diventare un piano alternativo al sistema vigente e come tale, per le autorità di governo, da affossare in blocco, e non solo da accantonare temporaneamente per il sopraggiungere del diluvio quarantottesco.

Col ritorno degli austriaci a Milano, infatti, ogni prospettiva di cambiamento fu annullata dal governo militare di Radetzki, durante il quale si restrinsero pure drasticamente gli spazi sia per le iniziative individuali che per le stesse attività istituzionali. Solo dopo l'arrivo dell'arciduca Massimiliano si poté parlare nuovamente di interventi graditi ai settori più dinamici della società, dalle opere pubbliche che erano da eseguire, all'attivazione di qualche nuovo insegnamento universitario, come quello libero di meccanica che nel ' 56 fu affidato al docente di geometria descrittiva Giovanni Codazza ${ }^{18}$. Nel clima dei secondi anni Cinquanta furono possibili iniziative locali, come l'Esposizione bresciana del 1857, volte a favorire la ripresa delle attività produttive duramente colpite dalla pebrina e da altri malanni. Già nel '55 i bresciani avevano inviato prodotti naturali, artistici e industriali della provincia all'Esposizione universale di Parigi, ottenendo apprezzamenti per i loro

Commissione, v. C. Cattaneo, Scritti sull'educazione e sull'istruzione, a cura di L. Ambrosoli, Firenze, La Nuova Italia, 1963, pp. 74-152.

17 In merito alla formazione degli ingegneri, qui più volte evocata per il ruolo che la categoria fu chiamata a svolgere dai processi di modernizzazione dell'Ottocento, la commissione sostenne la necessità di una preparazione più solida e differenziata, in considerazione dello sviluppo delle tecnologie a base scientifica e delle loro estese applicazioni. Riprendendo la richiesta avanzata da Francesco Colombani sul "Politecnico" sin dal 1839, la commissione dell'Istituto lombardo si espresse in questi termini: "Le nuove industrie abbisognano di Ingegneri meccanici, i quali dovrebbero inoltrarsi nello studio della Fisica Matematica, della Geometria descrittiva e della Meccanica applicata, principalmente per ciò che riguarda la cognizione pratica dei materiali, delle macchine a vapore, delle strade ferrate e delle costruzioni industriali. Dovrebbero viemeglio addestrarsi nel Disegno delle macchine, dovrebbero frequentare le lezioni di Chimica applicata alle Arti e a compiere la loro istruzione gioverebbero alcune letture di Conteggio amministrativo, d'Economia pubblica e di Scienza sanitaria al proposito delle manifatture" (v. C. Cattaneo, Scritti sull'educazione e sull' istruzione, cit., p. 118). In termini simili si esprimeva L. Tatti, Sulla riforma degli studi tecnici nelle provincie lombardo-venete, "Annali universali di statistica", ago-set. 1849, p. 128.

18 Ricordando gli inizi della sua carriera di tecnologo Giuseppe Colombo poté pertanto affermare molti anni dopo che quando fu chiamato a insegnare presso la Società d'incoraggiamento d'arti e mestieri di Milano dovette "imparare tutto" per proprio conto (Scritti e discorsi, vol. III, a cura di G. Gallavresi, Milano, Hoepli, 1934, p. 326). 
articoli in ferro. L'anno successivo la Camera di commercio, l'Ateneo bresciano e l'Amministrazione comunale puntarono all'allestimento dell'Esposizione industriale della provincia, che, per i patrioti liberali come Zanardelli divenne l'occasione per un approfondimento della realtà socio-economica della provincia e dei problemi da affrontare e risolvere ${ }^{19}$.

Come molti altri giovani lombardi che si erano avvicinati al "Crepuscolo" di Carlo Tenca, Zanardelli era ormai passato dalla critica a Carlo Alberto per il comportamento tenuto durante le vicende quarantottesche, al sostegno verso il nuovo Piemonte e la strategia cavouriana orientata sia alle mete risorgimentali dell'indipendenza e dell'unità, sia a una modernizzazione integrale del paese.

Se da una parte, infatti, Cavour favoriva il libero confronto delle idee e il dispiegarsi delle iniziative individuali (varò anche una nuova legge sui brevetti per incentivare le innovazioni), dall'altra era impegnato a creare le precondizioni dello sviluppo, potenziando il porto di Genova, estendendo l'irrigazione del Vercellese e del Novarese, costruendo le linee principali della rete ferroviaria e telegrafica, nonché a favorire la nascita delle officine meccaniche e cantieristiche richieste dall'ampio programma infrastrutturale, che includeva opere mai tentate prima, come il traforo alpino del Fréjus. Una modernizzazione insomma, quella perseguita dallo statista torinese, che, per ardimento e organicità (sostenne anche l'Esposizione di Genova del 1854 in occasione dell'apertura della ferrovia tra Torino e il capoluogo ligure) era in grado di conquistare le forze dinamiche della vicina Lombardia (come delle altre regioni italiane), che non avevano tardato a rimettersi in moto, come dimostrano la fondazione del "Crepuscolo", all'inizio degli anni Cinquanta, la rapida ripresa delle attività da parte della Società d'incoraggiamento ${ }^{20}$, e il tentativo di un gruppo di giovani intellettuali milanesi di ridare vita al "Politecnico" di Cattaneo e di riaggregare le forze progressiste all'insegna della cultura "positiva" ${ }^{21}$. Il tentativo non andò

19 Cfr. S. Onger, Verso la modernità. I bresciani e le esposizioni industriali 18001915, Milano, Angeli, 2010.

20 Cfr. Un inedito di Antonio Allievi, a cura di C.G. Lacaita, in "Rivista milanese di economia", 1989, fasc. 32, pp. 123-138.

21 Cfr. Da "Il Politecnico" di Cattaneo al Politecnico di Brioschi, a cura di A. Galbani e A. Silvestri, Milano, Politecnico di Milano, 2003, e "Il Politecnico" di Carlo 
in porto allora, ma è ugualmente una conferma del ruolo che gli ambienti risorgimentali lombardi assegnavano alle conoscenze scientifiche e tecnico-applicative in funzione dell'inserimento italiano nel processo di modernizzazione, di cui l'Esposizione universale di Londra del 1851 aveva mostrato a tutti le portentose realizzazioni ${ }^{22}$.

Ad affermarsi negli anni Cinquanta come elemento di raccordo fra i cultori delle scienze pure e quelli delle scienze applicate fu più di tutti Francesco Brioschi, matematico e ingegnere, attento ai diversi aspetti della ricerca e della formazione, ma anche ai loro risvolti pratici, e proprio per questo destinato ad assumere un ruolo centrale nella vita culturale italiana postunitaria ${ }^{23}$. Dopo aver cercato di rilanciare il "Politecnico" insieme a Gaetano Strambio e Emilio Cornalia, Brioschi si unì con altri matematici e docenti universitari, quali Enrico Betti (Pisa), Angelo Genocchi (Torino), Placido Tardy (Genova), e Barbana Tortolini (Roma), per dare impulso al rinnovamento della cultura scientifica italiana mediante gli "Annali di matematica pura e applicata”. Quindi, insieme a Betti e a Felice Casorati, allora giovane assistente a Pavia, intraprese nel '58 un lungo viaggio in Europa per presentare la nuova rivista, stabilire contatti con esponenti della ricerca d'oltralpe e conoscere da vicino gli ordinamenti e il funzionamento delle più rinomate istituzioni scientifiche e tecniche ${ }^{24}$. Il che gli consentì nel '59, conclusasi la seconda guerra d'indipendenza e iniziata, dopo le prime annessioni al Piemonte, la costruzio-

Cattaneo. La vicenda editoriale, i collaboratori, gli indici, a cura di C.G. Lacaita, R. Gobbo, E.R. Laforgia, M. Priano, Lugano-Milano, G. Casagrande editore, 2005.

22 Chiamato a far parte della Commissione internazionale per l'assegnazione dei premi, Luigi De Cristoforis, membro dell'Istituto lombardo e rappresentante della Camera di Commercio milanese, scrisse nella sua relazione che di fronte a tanti prodotti industriali si sentì "sopraffatto" dalla "somma delle cognizioni e degli sforzi umani qui rappresentati” (L. De Cristoforis, Relazione letta alla Camera di commercio e d'industria della provincia di Milano, Milano, 1852, p. 18). Sull'Esposizione londinese v. L. Aimone, C. Olmo, Le esposizioni universali, 1851-1900. Il progresso in scena, Torino, Allemandi, 1990, e P. Colombo, Le esposizioni universali: $i$ mestieri d'arte sulla scena del mondo (1851-2010), Venezia, Marsilio, 2012.

23 Sulla complessa figura del fondatore dell'Istituto tecnico superiore o Politecnico di Milano v. i contributi riuniti in Francesco Brioschi e il suo tempo (18241897), I, Saggi, a cura di C.G. Lacaita e A. Silvestri, Milano, Angeli, 2000.

24 Ne ricavò la memoria Della istruzione superiore in alcuni Stati d'Europa, apparsa in "La Perseveranza", 26 e 28 settembre 1863, ora in F. Brioschi, Scritti e discorsi, a cura di C.G. Lacaita, Franco Angeli, Milano, 2003, pp. 23-54. 
ne del nuovo sistema formativo italiano, di influire, insieme a Quintino Sella, sulle scelte del governo torinese in fatto di organizzazione degli studi superiori. Fu lui a far inserire nella Legge Casati del 1859 l'impegno ad attivare, accanto alla nuova Scuola di Applicazione per ingegneri di Torino, l'Istituto tecnico superiore di Milano (presto chiamato dai milanesi Politecnico), come vertice del secondo canale formativo, costituito dalle Scuole tecniche e dagli Istituti tecnici, che doveva nascere accanto a quello già esistente delle scuole classiche. In qualità di segretario generale della Pubblica Istruzione dal $1^{\circ}$ luglio 1861 al 17 gennaio 1863, Brioschi poté inoltre dare un forte impulso alla realizzazione della svolta postunitaria e del disegno che la ispirava ${ }^{25}$.

4. A organizzare un evento espositivo nazionale e un congresso straordinario degli scienziati italiani per festeggiare degnamente la nascita del nuovo Regno d'Italia, si impegnarono i fiorentini per le ragioni che di lì a qualche anno avrebbero portato Firenze a diventare la seconda capitale del Regno. Le due manifestazioni non mancarono di alimentare l'euforia patriottica del momento. Ma al netto dell'enfasi che si riversò nelle cronache, le due manifestazioni non tardarono a far riflettere. Il congresso, che doveva rivedere i regolamenti e adeguarli alla nuova situazione, non ebbe la partecipazione di "dotti" che gli organizzatori si attendevano. Né la successiva riunione, che si tenne nel settembre del 1862 a Siena, a quattordici anni di distanza da quella rinviata nel 1848, riuscì, ad avere un'affluenza paragonabile a quella dei congressi del primo periodo. Si dovette pertanto concludere che la partecipazione massiccia alle riunioni prequarantottesche apparteneva a una stagione che si era ormai conclusa. E non solo perché la tensione risorgimentale, che aveva richiamato a quelle riunioni generali centinaia di "dotti" per quindici giorni, aveva raggiunto le principali mete dell'indipendenza e dell'unità, ma anche perché si era entrati in una nuova fase dello sviluppo scientifico e del processo di modernizzazione a cui il fenomeno dei congressi era ugualmente legato. Anche gli altri due congressi postunitari, infatti, l'uno convocato a Roma per il 1873 e l'altro a Palermo per il 1875 , non fecero che confermare il cambiamento maturato dalla metà del secolo in poi.

25 Sulla svolta v. Le Università e l'Unità d'Italia, a cura di A. Ferraresi e E. Signori, Bologna, Clueb, 2011. 
La crescente specializzazione dei saperi e delle rispettive applicazioni tecnologiche spingevano sempre più verso forme di organizzazione e di comunicazione specifiche e differenziate, com'era chiaramente provato dalla nascita in Europa di molti nuovi centri di ricerca sia all'interno che al di fuori delle università. Era la stessa ragione che aveva spinto Brioschi, buon conoscitore del "movimento scientifico europeo", a volere per Milano un Istituto tecnico superiore simile ai politecnici dell'area germanica, cioè completo, autonomo e con sezioni diversificate al suo interno per essere adeguati alla crescita di nuove aree disciplinari tanto in campo tecnologico quanto in quello scientifico.

Legata al cambiamento in corso è anche la vicenda del "Politecnico" di Cattaneo, che giunto, dopo alcuni passaggi intermedi, in mano a Brioschi alla fine del 1865 , fu prima organizzato in due fascicoli distinti (fino al '68), uno dedicato ai temi "scientifico-letterari", l'altro a quelli tecnico-applicativi; successivamente, dal 1869 in poi (fusosi col "Giornale dell'ingegnere architetto" pubblicato dal '53 a Milano sotto la direzione di Raffaele Pareto), si concentrò sui temi delle scienze applicate, avendo come referenti e lettori i nuovi ingegneri italiani formatisi nelle scuole di applicazione postunitarie.

Quanto all'Esposizione fiorentina del 1861, prima rassegna nazionale dei prodotti italiani, se l'entusiasmo del momento non mancò di alimentare "il concerto di lodi", lo sguardo analitico di un esperto come Colombo non poté non rilevare che in realtà, a Firenze, si era potuto mostrare ben poco di simile a ciò che si produceva nei paesi più sviluppati ${ }^{26}$. Tra i segni dell'arretratezza complessiva, di cui si doveva prendere coscienza rapidamente, ricordava l'assenza negli stessi organizzatori di un criterio selettivo adeguato alla funzione propria delle esposizioni industriali moderne, attestata dalla presenza ingombrante di "tutti quei piccoli modelli di motori impossibili" che si sarebbero dovuti escludere in partenza dalla rassegna $a^{27}$.

Dopo aver fatto notare che delle molte locomotive ferroviarie operanti nella penisola, solo poche erano un prodotto nazionale, e che

26 Il lungo resoconto che Colombo scrisse per la "Perseveranza" apparve in otto puntate dal 13 ottobre al 29 dicembre. Ora è in Industria e politica nella storia d'Italia. Scritti scelti: 1861-1916, a cura di C.G. Lacaita, Milano-Roma Bari, CariploLaterza, 1985, pp. 89-152.

27 Ibidem, p. 114. 
ugualmente insignificante era "la quantità delle macchine di navigazione" fornite dalle officine italiane alle imprese del settore e alla marina nazionale, Colombo passò a sostenere la sua tesi di fondo, e cioè che, di fronte alla superiorità delle industrie estere già molto favorite dalla disponibilità di capitali, di materie prime e di risorse energetiche, era necessario e urgente un intervento pubblico guidato dalla consapevolezza delle difficoltà iniziali e dall'urgenza, imposta dalla stessa indipendenza nazionale, di disporre di officine meccaniche e di grandi cantieri, pronti a contribuire, se necessario, all" "armamento del paese" 28.

Da quella prima esperienza espositiva nazionale emerse pure in modo chiaro che, avendo tante cose da imparare e poche risorse da destinare, non era il caso per il momento di allestire altri eventi simili, e che conveniva piuttosto partecipare seriamente alle grandi esposizioni internazionali, che permettevano di apprendere dalle realtà più avanzate e insieme di farsi conoscere "dalle intraprese e dai capitali" esteri.

La prima occasione in tal senso fu offerta dall'Esposizione universale di Londra del 1862, e il governo nazionale si dedicò con tale impegno nella mobilitazione degli operatori interessati, che l'Italia risultò al secondo posto per numero di espositori, dopo l'Inghilterra, e al quarto posto per numero di premiati, dopo l'Inghilterra, la Francia e la Germania ${ }^{29}$.

Consapevole che dai paesi più evoluti occorreva mutuare non solo mezzi e pratiche di produzione ma anche strutture e metodi formativi, il governo recepì la proposta avanzata dalla delegazione guidata da Giuseppe Devincenzi, e deliberò la creazione a Torino del Museo industriale italiano, sull'esempio del Kensington Museum esistente a Londra e di altre istituzioni europee come il Conservatoire des Arts et Métiers di Parigi.

Per dare inoltre risalto al tema del "risorgimento economico" come completamento di quello "politico", furono organizzate pubbliche cerimonie in occasione della consegna delle medaglie e dei diplomi ottenuti dagli espositori italiani. Nel discorso pronunciato a Milano Giuseppe Colombo indicò tra le priorità da perseguire una moderna organizzazione del lavoro, un'efficace diffusione dei "risultati della scienza" tra "i fabbricanti e gli operaj", un'adeguata valorizzazione nelle produzioni nazionali dell'“elemento estetico" così fortemente

28 Ibidem, pp. 91, 105, 112.

29 Ibidem, p. 154. 
radicato nel gusto degli italiani, e che a giudizio del tecnologo milanese era destinato ad avere un rilievo sempre maggiore nelle società evolute. "Col crescere della prosperità", argomentava, anche le "abitudini all'eleganza si diffondono" in larghi strati di popolazioni ${ }^{30}$.

A confermare l'importanza della formazione e della cultura per la nuova Italia in affanno per i tanti problemi nazionali, e in ritardo rispetto all'avanzamento dei paesi più moderni, giunse di lì a poco l'Esposizione di Parigi del 1867, che aprì un ampio dibattito sui rapporti sempre più stretti di scienza, tecnica e attività produttive, $\mathrm{e}$ sul ruolo assunto dai sistemi formativi nei processi di industrializzazione.

Per la prima volta, infatti, era avvenuto che, a ricevere più riconoscimenti anche nei settori tecnologicamente cruciali, non fosse più la prima potenza industriale, come era stato fino allora, ma i principali paesi inseguitori. Ne derivò un ampio dibattito, al di qua e al di là della Manica, e molti conclusero che la ragione della rimonta dei second comers andava ricercata nella superiorità dei sistemi continentali d'istruzione tecnico-scientifici e professionali realizzati dai pubblici poteri, rispetto al sistema inglese "di self-instruction", rimasto legato all'iniziativa individuale in nome dei principi liberisti posti alla base dei rapporti fra capitale e lavoro ${ }^{31}$.

Per la nuova classe dirigente italiana l'Esposizione parigina fu una conferma del paradigma risorgimentale che aveva ispirato le maggiori novità della Legge Casti, e al tempo stesso un forte incitamento ad andare avanti nella costruzione di un'ampia rete di nuove strutture formative.

Così, mentre erano ancora in rodaggio le istituzioni decise nel '59 per Torino e per Milano, e mentre si riorganizzavano le già esistenti Scuole per gli ingegneri di Napoli e di Padova, si proseguì nella costruzione del nuovo sistema formativo, aggiungendo via via la Scuola superiore di commercio di Venezia (1867), quella navale di Genova (1870) e quella mineraria di Palermo (1872), le due Scuole di agricoltura di Milano (1870) e di Portici (1872), e quella di ingegneria già esistente a Roma, nonché le altre di vario livello e indirizzo, dall'Istituto forestale di Vallombrosa (1869) alla Stazione sperimentale di caseificio di Lodi

30 Ibidem, pp. 160-161.

31 Ibidem, pp. 167-177. 
(1871), a quella bacologica di Padova (1871), alle altre finalizzate alla viticoltura, alle attività nautiche e di pesca, alle miniere, fra cui le scuole di Caltanisetta (1862) e Iglesias (1871).

Le tante nuove strutture formative però non sostituirono, ma si aggiunsero agli organismi accademici e scolastici più asfittici ereditati dal passato, nonostante le proposte e i tentativi che pure furono fatti in tal senso. Fra gli stessi fautori delle nuove scuole non mancò quindi chi sostenne di chiudere anche su questo versante le meno solide per consentire ai tre principali centri politecnici (Milano, Torino e Napoli) di poter competere con le grandi istituzioni europee ${ }^{32}$ La messa in equilibrio del sistema sarà pertanto lenta e sostanzialmente legata al superamento delle emergenze nazionali e alla possibilità degli enti locali di intervenire tramite appositi consorzi a sostegno delle istituzioni che desideravano mantenere in vita.

5. Con la conquista del Lazio e la proclamazione di Roma capitale era intanto giunto a compimento il processo di unificazione della penisola, e tra le manifestazioni da organizzare non mancarono le "feste del progresso", com'erano chiamate le esposizione industriali. Ad allestire le due rassegne del 1871 provvidero sia Torino, che aveva da celebrare anche l'apertura del Fréjus, sia Milano che era la punta avanzata della modernizzazione italiana. Ma per la brevità del tempo trascorso dall'entrata in campo dello Stato nazionale, non si potè ralizzare che manifestazioni ridotte. $\mathrm{E}$ infatti, se la rassegna torinese risultò una mostra "campionaria" collocata nei ristretti spazi del Museo industriale italiano con la partecipazione di 511 espositori, due terzi dei quali piemontesi ${ }^{33}$ ), l'esposizione milanese, promossa dall'Associa-zione industriale italiana, fu sì più ampia, contando 1190 operatori (di cui più della metà di Milano e solo 370 di altre parti d'Italia), ma, presentandosi concentrata sui prodotti tessili, alimentari, per l'edilizia e della meccanica corrente, non mostrò innovazioni rilevanti dal punto di vista tecnologico.

Fu negli anni Settanta peraltro che, per lo shock prodotto dall'in-

32 D. Padelletti, Le scuole politecniche d'Italia e di Germania con speciale riguardo all'insegnamento della meccanica, "Nuova Antologia", 1974, pp. 679-680.

33 Le esposizioni torinesi 1805-1911. Specchio del progresso e macchina del consenso, a cura di U. Levra, R. Roccia, Torino, Archivio storico, 2003. 
felice prova italiana nella guerra del ' 66 contro l'Austria, e per la grande inchiesta del 1870-74 sulle attività industriali, non solo si prese coscienza delle insufficienze da colmare, ma cominciò a cambiare anche la politica economica e doganale (1878) fino allora ispirata al libero-scambismo iniziale. Sempre in quegli anni inoltre, l'apparato produttivo del futuro triangolo industriale cominciò a utilizzare le nuove opportunità offerte dall'unificazione e a dare chiare prove di ripresa, specie dove esistevano le premesse più favorevoli, come a Milano, e più incisiva era anche l'azione delle nuove strutture formative.

Qui infattil'Istituto tecnico superiore, dopo l'avvio e i primi anni di assestamento, iniziò ad affermarsi, sotto la ferma guida di Brioschi, come polo efficace di cultura tecnico-scientifica e come centro formativo di nuovi talenti imprenditoriali. Quando si scorre l'elenco dei primi laureati milanesi non si può non rilevare che tanti protagonisti dell'industrializzazione postunitaria, da Bartolomeo Cabella a Giulio Prinetti, da Alberto Riva ad Aristide Rubini, da Cesare Saldini a Giuseppe Ponzio, si formarono nel Politecnico milanese, studiando e lavorando insieme a docenti capaci di avviarli su sentieri imprenditoriali nuovi. È il caso, per fare solo due esempi, sia della Filotecnica, industria meccanica di precisione e di strumenti ottici fondata nel 1865 da Ignazio Porro, docente di celerimensura, e poi passata ad Angelo Salmoiraghi, uno dei primi laureati milanesi in ingegneria industriale ${ }^{34}$, sia della Pirelli e C. per la lavorazione della gomma, avviata nel 1872 su consiglio di Colombo e col sostegno finanziario procurato da Brioschi e da altri ${ }^{35}$.

Ancor più incisiva divenne l'azione del Politecnico quando poté attivare il biennio propedeutico interno (1875) col contributo degli enti locali, e ampliare l'offerta didattica grazie sia alle intese con le altre istituzioni culturali milanesi, sia al sostegno del mondo produttivo, che consentì l'inserimento di nuovi insegnamenti, come quello di

34 C.G. Lacaita, Ignazio Porro e le origini dell'industria ottico-meccanica di precisione, "Rivista milanese di economia", n. 36, 1990, pp. 118-130.

$35 \mathrm{Al}$ giovane Pirelli, che dopo la laurea era stato mandato in Europa con una borsa di studio per completare la sua formazione a contatto diretto con diverse realtà industriali, Colombo raccomandava nel marzo del ' 71 di osservare vari processi tecnico-produttivi, ma di non perdere di vista "l'obiettivo del Caoutchouc". Rientrato in patria G.B. Pirelli si dedicò alla fondazione della nuova industria con capitali procurati dai maestri. 
Economia industriale finanziato da Eugenio Cantoni nel 1871, e l'incremento di un settore di punta com'era allora l'elettrotecnico in rapida espansione.

È del 1877 la prima prova di illuminazione pubblica elettrica con una potente lampada ad arco posta in cima ad una torre eretta in piazza del Duomo. Il passo successivo fu compiuto nel giugno del 1881 con l'illuminazione della Galleria Vittorio Emanuele, quando furono accese 25 lampade ad arco della Siemens, per una potenza complessiva di 20.000 candele. L'evento fu organizzato in occasione della grande Esposizione Nazionale allestita nell'area dei Giardini pubblici, con la quale Milano intese mostrare finalmente gli esiti concreti del ventennale cammino postunitario. Per la prima volta a guidare gli organizzatori dell'esposizione c'erano gli uomini del Politecnico che contribuirono a rendere rigorosa la selezione dei prodotti da esporre, e ancor più a fare dell'Esposizione un momento di collettiva presa di coscienza dei problemi aperti in tema di sviluppo, mediante articoli, libri, conferenze, convegni e pubbliche manifestazioni.

Sia per la quantità degli espositori (7.139) e dei visitatori (oltre un milione) provenienti da ogni parte d'Italia, anche se maggiormente dalle regioni settentrionali, sia per le novità che poté presentare e per l'interesse che riuscì a suscitare ad ampio raggio attraverso la stampa, quella dell'81 fu certamente l'Esposizione di maggiore rilievo fino allora realizzata in Italia. E da allora ha continuato, non a caso, a rappresentare una tappa fondamentale nel processo di modernizzazione italiano.

Illustrando le novità che avevano reso l'esposizione milanese una "sorpresa", Colombo non mancò di rilevare quella che, a suo avviso, costituiva una delle principali ragioni dell'effettivo cambiamento registrato nell'81, e che portava a guardare con ottimismo al futuro del paese. Tale ragione stava nella maggiore "coltura scientifica del personale dirigente" delle nuove industrie, e in particolare nella "schiera di giovani istruiti e valorosi" che, formatisi nelle scuole politecniche postunitarie, si erano già inseriti nella società e nel tessuto produttivo come agenti di sviluppo e di innovazione. In un'epoca in cui "tutto si fa e si calcola colle regole" date dalla "teoria, sussidiata dall'esperienza", argomentava Colombo, i costruttori e gli imprenditori italiani, dominati fino allora dal "più volgare empirismo", si erano finalmente convinti "che senza il sussidio dell'elemento scientifico era preclusa la via a qualunque miglioramento". E per chi, come lui, si era dedicato all'insegnamento delle moderne tecnologie, costituiva un motivo di vivo compia- 
cimento il constatare che "una non piccola parte dei progressi manifestati dalla esposizione attuale" era dovuta proprio agli allievi delle nuove scuole politecniche, diventati ormai o direttori o gerenti o fondatori di stabilimenti vitali ${ }^{36}$.

Che in effetti si fossero create le condizioni per l'evoluzione successiva, emerse subito dopo l'81 col pronto inserimento dei centri della cultura tecnico-scientifica italiani e dei suoi uomini, i Colombo e i Ferraris, nello sviluppo dell'elettrotecnica e dell'industria elettrica, che tanto profondamente avrebbe segnato la nuova fase del processo ottocentesco di industrializzazione ${ }^{37}$. Significativamente, mentre già dall'82 il Politecnico milanese provvedeva ad attivare un insegnamento speciale sulle macchine dinamo-elettriche affidandolo a Ferrini, sia Colombo sia Ferraris, dopo aver partecipato all'Esposizione parigina dedicata all'elettrotecnica (1881), diedero un contributo decisivo al cambiamento che si era appena avviato: Colombo realizzando a Milano la prima applicazione continentale del sistema di illuminazione Edison alimentato da una centrale termoelettrica (1883); Ferraris sviluppando le ricerche che lo portarono a scoprire il campo magnetico rotante (1882) e poco dopo a ideare il motore elettrico a corrente alternata.

Per partecipare come paese, e non come individui particolarmente dotati, alla nuova fase dello sviluppo, caratterizzata ben più della precedente dall'accelerazione delle scoperte e dall'intreccio di scienza, tecnica e attività produttive, era necessario disporre a tutti i livelli, in particolare a quelli più elevati, di una preparazione adeguata e sufficientemente diffusa. Il che, nonostante le difficoltà e i limiti di sopra ricordati, fu possibile grazie alle nuove istituzioni formative e di ricerca create subito dopo la svolta unitaria.

36 G. Colombo, Industria e politica nella storia d'Italia, cit., pp. 256-257.

37 Storia dell'industria elettrica in Italia, I. Le origini. 1881-1914, a cura di G. Mori, Roma-Bari, Laterza, 1992. 